\title{
Stabilization and precise calibration of a continuous-wave difference frequency spectrometer by use of a simple transfer cavity
}

\author{
E. Riedle, ${ }^{\text {a),b) }}$ S. H. Ashworth, ${ }^{\text {b) }}$ J. T. Farrell, Jr., and D. J. Nesbitt ${ }^{c}$ \\ Joint Institute for Laboratory Astrophysics, University of Colorado and National Institute of Standards \\ and Technology, Boulder, Colorado 80309-0440
}

(Received 15 July 1993; accepted for publication 24 September 1993)

\begin{abstract}
A novel, simple, and inexpensive calibration scheme for a continuous-wave difference frequency spectrometer is presented, based on the stabilization of an open transfer cavity by locking onto the output of a polarization stabilized HeNe laser. High frequency, acoustic fluctuations of the transfer cavity length are compensated with a piezoelectric transducer mounted mirror, while long term drift in cavity length is controlled by thermal feedback. A single mode $\mathrm{Ar}^{+}$laser, used with a single mode ring dye laser in the difference frequency generation of $2-4 \mu \mathrm{m}$ light, is then locked onto a suitable fringe of this stable cavity, achieving a very small long term drift and furthermore reducing the free running $\mathrm{Ar}^{+}$linewidth to about $1 \mathrm{MHz}$. The dye laser scan provides tunability in the difference frequency mixing process, and is calibrated by marker fringes with the same stable cavity. Due to the absolute stability of the marker cavity, precise frequency determination of near infrared molecular transitions is achieved via interpolation between these marker fringes. It is shown theoretically that the residual error of this scheme due to the dispersion of air in the transfer cavity is quite small, and experimentally that a frequency precision on the order of $1 \mathrm{MHz}$ per hour is routinely obtained with respect to molecular transitions.
\end{abstract}

\section{INTRODUCTION}

Over the past decade, continuous-wave (cw) laser spectrometers with $\mathrm{MHz}$ levels of spectral resolution have been developed over the near ultraviolet (UV),${ }^{1-3}$ all of the visible ${ }^{4,5}$ and large regions of the infrared. ${ }^{6-8}$ In these subDoppler molecular spectrometers full spectroscopic use of the extremely high resolution can only be made if a suitable calibration scheme is implemented. Since the scanning of the spectrum typically takes hours, excellent long term stability of the system is necessary.

One way to obtain the proper calibration is by comparison to a precisely known reference spectrum. However, such spectra are often not available in all regions of interest and can be difficult to record simultaneously. A second way to obtain precise spectral information for various molecular lines is by direct measurement of the wavelength or frequency in a wavemeter (traveling Michelson interferometer). It has been shown ${ }^{9}$ that this is possible with an absolute precision of better than $1 \mathrm{MHz}$ but for most systems this is prohibitively time consuming.

The most frequently used calibration method is therefore by interpolating the molecular spectrum with the transmission spectrum of a suitable Fabry-Perot cavity. No absolute calibration is normally attained in this way, but for most applications, a relative calibration is easily obtained and quite sufficient. On the other hand, the abso-

\footnotetext{
a) 1991-92 JILA Visiting Fellow.

b) Present address: Max-Born-Institut für Nichtlineare Optik und Kurzzeitspektroskopie, Rudower Chaussee 6, D-12489 Berlin, Germany.

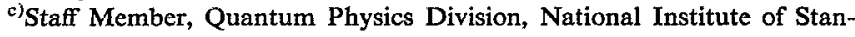
dards and Technology.
}

lute accuracy can be quite high if it is possible to keep the optical spacing of the mirrors constant. This is typically done by using a low thermal expansion spacer like Zerodur ceramic and high quality temperature control. ${ }^{10,11}$ In addition the cavity has to be evacuated to avoid any drift of the transmission fringes due to change in the refractive index in the cavity caused by ambient pressure changes.

The interest in a calibration precision on the order of 1 $\mathrm{MHz} / \mathrm{h}$ is quite keen and no commercial systems are readily available. We therefore have set out to design and build a simple system for use with our difference frequency spcctrometer that can perform to this level of accuracy. Instead of the more conventional reliance on the long term thermal stability of a Zerodur material, we have opted to lock an open cavity actively to a polarization stabilized $\mathrm{HeNe}$ laser and use this as a transfer cavity for the frequency stabilization of the $\mathrm{Ar}^{+}$laser used in conjunction with a single mode dye laser to generate the infrared (IR) light. In addition, this cavity is used to record marker fringes from the scanning of the dye laser, which therefore provides accurate frequency markers in the infrared. The design of the system will be described in detail below and the magnitude of possible drifts due to dispersion of air discussed. Finally, the successful performance of the instrument is demonstrated by recording a known molecular spectrum repeatedly over many hours and comparing the successive frequency measurements.

\section{DESIGN OF THE SYSTEM}

\section{A. Basic considerations}

The conventional approach to the construction of a highly stable calibration setup for optical spectrometers is 
the use of a suitable Fabry-Perot interferometer (FPI). A fixed frequency laser can either be locked to one of the cavity modes of the FPI or it can be used for calibration of a tunable laser scan. To reduce the influence of ambient temperature and pressure changes, a spacer for the mirrors with very low expansion coefficient is preferred and the whole optical cavity is typically placed in a sealed tank. ${ }^{10,11}$ The tank is often evacuated and needs to be kept at as constant a temperature as possible.

To understand the necessary demands on the stability of the temperature of such a system and the tolerable pressure changes, we start from the well known condition for the frequency $v$ of a given longitudinal mode of a confocal FPI

$$
y=\frac{N c_{0}}{4 n d},
$$

with $d$ the spacing between the mirrors, $c_{0}$ the speed of light in vacuum, $n$ the refractive index of the medium between the mirrors, and $N$ the integral mode number. For the $632.8 \mathrm{~nm}\left(4.7361 \times 10^{14} \mathrm{~Hz}\right)$ output wavelength of a HeNe laser, the refractive index of air under standard conditions of 760 Torr $\left(1\right.$ Torr $=133 \mathrm{~Pa}$ ) pressure $p_{0}$ and 288 $\mathrm{K}$ temperature $T_{0}$ is approximately $n=1+2.7652 \times 10^{-4}$, where the quantity $(n-1)$ is linearly proportional to the density of the gas medium. ${ }^{12}$ A change $\delta p$ in pressure at constant temperature will lead to a change of the density of the air in the cavity, and consequently to a new frequency $v+\delta v$ of the desired mode given by

$$
v+\delta v=\frac{N c_{0}}{4\left[n+(n-1)\left(\delta p / p_{0}\right)\right] d} \approx \frac{N c_{0}}{4 n d}\left(1-\frac{n-1}{n} \frac{\delta p}{p_{0}}\right) .
$$

From Eqs. (1) and (2) it follows that

$$
\delta v \approx-\frac{n-1}{n} \frac{\delta p}{p_{0}} v \approx-(n-1) \frac{\delta p}{p_{0}} v,
$$

which the HeNe laser frequency at near ambient pressures amounts to a $\delta v / \delta p$ of $-172 \mathrm{MHz} / \mathrm{T}$ orr. Therefore, the pressure of the air between the mirrors has to be controlled to better than 0.01 Torr if a stability of the FPI on the order of $1 \mathrm{MHz}$ is to be achieved. Hence, the FPI is typically placed inside a vacuum tank, though this is explicitly not the choice made for the transfer cavity design presented herein.

The influence of a temperature change on an open cavity is twofold. On the one hand, it will change the density of the air in the FPI and influence the frequency of the cavity mode in a similar fashion to a pressure change, and on the other hand the physical spacing of the mirrors will be changed by thermal expansion of the spacer. The former effect will be considered first.

At constant pressure, a temperature change $\delta T$ leads to a density change of the air between the mirrors which in turn leads to a frequency change of the cavity mode of

$$
\delta v \approx(n-1) \frac{\delta T}{T_{0}} v
$$

where $n$ is the index at temperature $T_{0}$. As an example, for the $\mathrm{HeNe}$ laser at atmospheric pressure this already amounts to a $\delta v / \delta T$ of $455 \mathrm{MHz} / \mathrm{K}$. Hence at ambient pressure, even small temperature changes would cause significant drift. Again, keeping the FPI in an evacuated tank can eliminate this density related dependence of the mode frequency on the temperature.

The more commonly treated temperature influence on the frequency of a given FPI cavity mode is the thermal expansion of the spacer used to separate the mirrors. For a thermal expansion coefficient $\alpha$ of the spacer this infiuence can be seen to be

$$
v+\delta v=\frac{N c_{0}}{4 n d(1+\alpha \delta T)} \approx \frac{N c_{0}}{4 n d}(1-\alpha \delta T)
$$

or

$$
\delta v \approx-\alpha \delta T v .
$$

For Zerodur ceramic $\alpha=-0.3 \times 10^{-7} / \mathrm{K}$ and $\delta v / \delta T=14$ $\mathrm{MHz} / \mathrm{K}$, while for fused silica $\alpha=5.5 \times 10^{-7} / \mathrm{K}$ and $\delta v /$ $\delta T=260 \mathrm{MHz} / \mathrm{K}$. This means that even for extremely low expansion Zerodur as a spacer, the absolute temperature of the FPI must be controlled to better than $0.1 \mathrm{~K}$ to ensure a frequency stability on the order of $1 \mathrm{MHz}$.

\section{B. The FPI as a transfer cavlty}

The reasons discussed above commonly lead to the use of an actively temperature controlled FPI with a low expansion spacer placed in a vacuum tank if high precision calibration of optical spectra is needed. However, such a system is fairly complicated to build and not very convenient to align. The alternative we consider is an open cavity (at ambient pressure) which is not passively stabilized but instead, locked to a simple reference laser with a highly stable output frequency. Such a reference laser is indeed readily available in the polarization stabilized $\mathrm{HeNe}$ laser. ${ }^{13}$ What remains to be investigated is the locking scheme and how well such a transfer cavity system performs over ranges of wavelengths (frequencies) both close and far from the HeNe laser output.

The system we have devised both (i) to stabilize the frequency of the $\mathrm{Ar}^{+}$laser and (ii) to calibrate the scan of the dye laser used for the near IR cw difference frequency generation in our spectrometer ${ }^{7}$ is shown schematically in Fig. 1. In the interest of simplicity, only the optical components are depicted.

The heart of the system is a $30 \mathrm{~cm}$ length confocal FPI with very broadband mirrors ( $T=6 \pm 2 \%$ for $400-780$ $\mathrm{nm})$. The measured finesse of this cavity is 20 , i.e., it has a fringe width of $13 \mathrm{MHz}$ at the $250 \mathrm{MHz}$ free spectral range (FSR). The cavity is locked onto the frequency of a polarization stabilized $\mathrm{HeNe}$ laser whose absolute frequency stability is measured to be on the order of $0.1 \mathrm{MHz} / \mathrm{h}$ by optical mode beating against an iodine stabilized $\mathrm{HeNe}$ laser. Slow control of the cavity length is performed by heating the middle section of the confocal mirror spacer, while fast control is obtained using a piezoelectric transducer (PZT)-supported end mirror. The $\mathrm{Ar}^{+}$laser fre- 


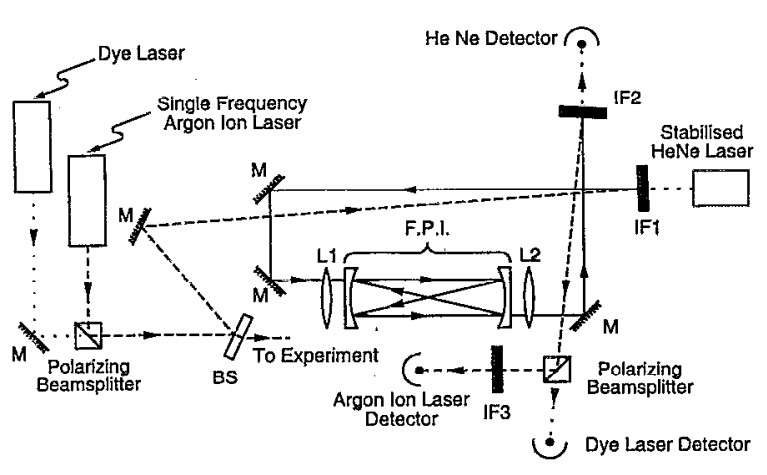

FIG. 1. Schematic diagram of the optical setup used for stabilization of a difference frequency spectrometer. The transfer cavity (FPI) is locked onto a polarization stabilized HeNe laser. The single frequency $\mathrm{Ar}^{+}$laser, in turn, is locked to the cavity. Finally the scan of the dye laser is calibrated by recording the transmission fringes of the stabilized transfer cavity. A number of mirrors (M) are used to steer the beams, interference filters (IF) and polarizing beamsplitters are employed to combine the various laser beams collinearly or separate them after passing through the cavity. Two planoconvex lenses ( $\mathrm{L} 1$ and $\mathrm{L} 2$ ) with $f=500 \mathrm{~mm}$ are used to compensate for the refractive power of the cavity mirrors. All three beams are monitored on separate detectors. A beamsplitter (BS) is used to select the required amount of dye laser and $\mathrm{Ar}^{+}$laser radiation out of the main beam going to the experiment.

quency is then locked to a suitable cavity mode of the FPI. Finally, the transmission pattern of the FPI is recorded for calibration as the dye laser is scanned. All three laser beams are suitably attenuated and combined collinearly with a polarizing beamsplitter and/or interference filter before they are coupled into the transfer cavity. The transmitted light is similarly split into its three components and each color detected on a separate detector.

The design of the FPI is shown in somewhat more detail in Fig. 2. Starting from the right, the spacer consists of a 12-cm-long section of 2.5 -cm-diam pyrex tubing, two stainless steel-to-pyrex graded seals welded together with their stainless steel sides, a second 8-cm-long section of pyrex, and finally a $2.5-\mathrm{cm}$-long tubular piezoceramic transducer (PZT). Most of the stainless steel part of the spacer is wrapped with heating tape to allow active control of the cavity length.

The numerical example given in the preceding section (HeNe laser frequency) shows that a 1 Torr increase in

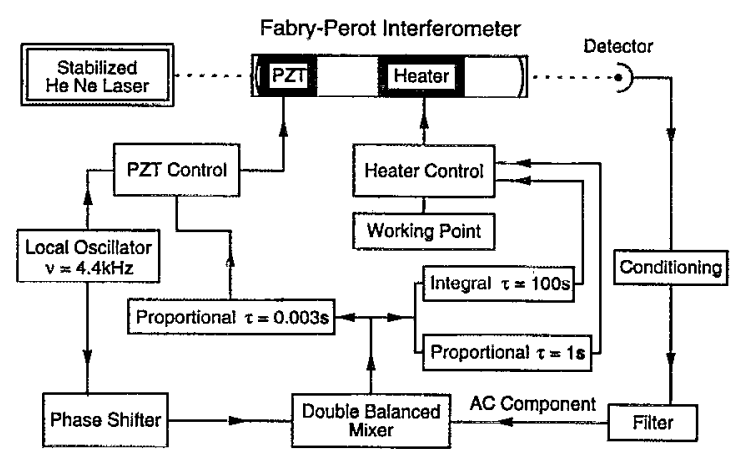

FIG. 2. Schematic diagram of the electronic locking scheme used to stabilize the transfer cavity onto the stabilized HeNe laser. See text for details. pressure (at constant temperature) of the air in the cavity would lead to a frequency shift of the cavity mode of -172 $\mathrm{MHz}$. If the whole length of the spacer were heated for thermal control of the cavity length and fused silica used for a spacer, a drop by $0.7 \mathrm{~K}$ would compensate for this pressure change. However, heating the whole length is not very practical, instead only a fifth can typically be heated. For a fused silica cavity, the temperature of this shorter section must now be changed by $3.5 \mathrm{~K}$. For a small pressure change this scaling might just be acceptable, for larger variations it turns out to be prohibitive. Hence we have chosen a 6-cm-long stainless steel section as the heated part of the spacer, i.e., a material with a much higher thermal expansion coefficient $\left(\alpha=187 \times 10^{-7} \mathrm{~K}\right)$, and for which a drop in temperature of only $0.1 \mathrm{~K}$ suffices to compensate for the 1 Torr pressure rise. This allows access to a wide dynamic locking range with only a moderate initial heating (about $20 \mathrm{~K}$ above room temperature). In addition, the effect of heating the air inside the spacer [e.g., see Eq.(4)] is minimized. Once the stainless steel part of the spacer has been chosen for the described reasons, pyrex glass with a five-fold smaller expansion coefficient $\alpha=32 \times 10^{-7} / \mathrm{K}$ is a natural and convenient choice for the remaining sections of the spacer. In particular, graded seals of stainless steel and pyrex are readily available and the five-fold ratio between the two expansion coefficients can nearly perfectly compensate for the five-fold ratio in section lengths. A $1 \mathrm{~K}$ change in temperature of the pyrex (e.g., by a change in the lab temperature) can therefore be compensated by an equal but opposite change of the temperature of the stainless steel. These opposing effects help minimize the influence of ambient temperature changes on the average temperature of the air inside the FPI and the associated frequency shifts from changes in air density.

\section{Possible limitations of performance}

The system described in the preceding section transfers the stability of the reference $\mathrm{HeNe}$ laser to both the $\mathrm{Ar}^{+}$ laser and the dye laser fringes through the use of an open transfer cavity. For this purpose, the length of the cavity is controlled so as to compensate for any changes in ambient pressure and temperature. However, the associated shift in frequency of any cavity mode depends on the refractive index of the medium between the mirrors at the corresponding wavelength. Since the refractive index of air depends upon wavelength, there will still be a small residual error in the system due to this dispersion that eventually limits the performance of the system. This section addresses at what level of precision this will happen. For simplicity, we consider only the pressure dependence (at constant temperature) but by comparison of Eqs. (3) and (4), the temperature dependence can be obtained by simple scaling.

To lock a cavity mode of the FPI (with mode number $\left.N_{\mathrm{HeNe}}\right)$ to the frequency $\left(v_{\mathrm{HeNe}}\right)$ of the stabilized $\mathrm{HeNe}$ laser at ambient pressure $(p)$, the length $(d)$ of the confocal cavity has to be 


$$
\begin{gathered}
d=\frac{N_{\mathrm{HeNe}} c_{0}}{4 v_{\mathrm{HeNe}}} \frac{1}{1+\left(n_{\mathrm{HeNe}}-1\right)\left(p / p_{0}\right)} \\
\approx \frac{N_{\mathrm{HeNe}} c_{0}}{4 v_{\mathrm{HeNe}}}\left(1-\left(n_{\mathrm{HeNe}}-1\right) \frac{p}{p_{0}}\right) .
\end{gathered}
$$

To compensate for a change $\delta p$ in pressure, the mirror spacing must be adjusted to $d+\delta d$, where

$$
\frac{\delta d}{\delta p} \approx \frac{-N_{\mathrm{HeNe}} c_{0}}{4 v_{\mathrm{HeNe}}}\left(n_{\mathrm{HeNe}}-1\right) \frac{1}{p_{0}}
$$

or

$$
\delta d \approx-d\left(n_{\mathrm{HeNe}}-1\right) \frac{\delta p}{p_{0}} .
$$

In Eq. (9) the refractive index was set equal to 1 since we consider only linear terms in $\delta d$, i.e., first order changes in the cavity length.

A second mode of the transfer cavity (the mode number $N_{\text {laser }}$ ) with a frequency close to either the $\mathrm{Ar}^{+}$laser or the dye laser will, by analogy with Eq. (7), have a frequency $v_{\text {laser }}$ at the initial pressure of

$$
v_{\text {laser }} \approx \frac{N_{\text {laser }} c_{0}}{4 d}\left(1-\left(n_{\text {laser }}-1\right) \frac{p}{p_{0}}\right) .
$$

With the pressure changed by $\delta p$ and the accompanying change in length $\delta d$ to keep the cavity locked to the HeNe, the frequency for the mode will be changed by $\delta v_{\text {laser }}$ according to

$v_{\text {laser }}+\delta v_{\text {laser }}=\frac{N_{\text {laser }} c_{0}}{4 d}\left(1-\frac{\delta d}{d}\right)\left(1-\left(n_{\text {laser }}-1\right) \frac{p+\delta p}{p_{0}}\right)$.

Substituting Eq. (9) and taking only linear terms in the changes and rearranging, one finally obtains

$$
\begin{aligned}
\delta v_{\text {laser }} & \approx \frac{N_{\text {laser }} c_{0}}{4 d} \frac{\delta p}{p_{0}}\left(n_{\mathrm{HeNe}}-n_{\text {laser }}\right) \\
& \approx v_{\text {laser }} \frac{\delta p}{p_{0}}\left(n_{\mathrm{HeNe}}-n_{\text {laser }}\right):
\end{aligned}
$$

Equation (12) shows that the residual error from the dispersion of the refractive index of air is proportional to the difference between the refractive index at the wavelength of the HeNe laser to which the cavity is locked and that of the second laser. Hence, the closer the two wavelengths can be chosen, the lower the residual error from the use of an open transfer cavity will be.

In our experiment the cavity is locked to the $632.8 \mathrm{~nm}$ line of the HeNe, and $3963 \mathrm{~cm}^{-1}$ IR light is generated by difference frequency mixing the $488.0 \mathrm{~nm} \mathrm{Ar}^{+}$line (20486 $\mathrm{cm}^{-1}$ ) and the output of the dye laser in the $605 \mathrm{~nm}$ region. The refractive index of air (under standard temperature and pressure conditions) at these two wavelengths is $1+2.7929 \times 10^{-4}$ and $1+2.7690 \times 10^{-4}$, respectively. ${ }^{12}$ With $n_{\mathrm{HeNe}}=1+2.7652 \times 10^{-4}$ this results in residual errors of $\delta v_{\text {dye }} / \delta p=-0.25 \mathrm{MHz} /$ Torr and $\delta v_{\mathrm{Ar}} / \delta p=-2.24$ $\mathrm{MHz} /$ Torr. For the resulting IR light, the drift is only $-1.99 \mathrm{MHz} / \mathrm{Torr}$. One can see that, for the small pressure changes encountered in a typical laboratory environment, a stability on the order $\leqslant 5 \mathrm{MHz}$ can be expected. Hence, the described transfer cavity scheme reduces the drift in calibration caused by pressure changes by about two orders of magnitude from the value expected for a simple marker cavity (compare Sec. II A).

As a final point, it should be mentioned that even though there will be a considerable change in the length of the transfer cavity if the pressure changes a few Torr, the stabilization of the cavity to the HeNe laser ensures that the free spectral range (FSR) changes by only an extremely small amount (on the order of 1 part in $10^{8}$ ). Therefore, the FSR has to be calibrated only once to obtain the proper scaling of the dye laser calibration fringes. A new calibration is only required if a sufficiently different mode of the transfer cavity is locked onto the HeNe laser.

\section{IMPLEMENTATION}

In this section we explain in more detail how the cavity is actually locked to the stabilized HeNe laser and the $\mathrm{Ar}^{+}$ laser in turn to the cavity. The recording of the transmission fringes with scanning of the dye laser is standard and will not be detailed.

\section{A. Locking of the transfer cavity to the HeNe laser}

The scheme of locking the transfer cavity to the HeNe laser is shown in Fig. 2. There are two ranges of frequency control needed: a fast response to correct for the small amplitude, predominately acoustic fluctuations and a slow but large amplitude response to correct for the changes in average pressure and temperature in the laboratory.

For determining the direction and magnitude of the servo loop correction, a derivative shaped "discriminator" signal is required. An elegant optical method to achieve this was reported recently; ${ }^{14}$ it does, however, require additional optical components not compatible with the triple laser setup used in this work. Instead, to obtain an error signal, the following scheme is used. The PZT is driven by a small sinusoidal voltage $\left(V_{p p} \approx 0.125 \mathrm{~V}\right)$ derived from a local oscillator running at a frequency of $v_{\text {mud }}=4.4 \mathrm{kHz}$. The PZT changes the transmission frequency of the FPI by one free spectral range (FSR) every $35 \mathrm{~V}$. This small dither voltage therefore introduces a cavity modulation of $\$ 1 \mathrm{MHz}$ (i.e., less than $10 \%$ of the transmission width of a fringe) which is detected as amplitude modulation of the transmitted light at the frequency $v_{\text {mod }}$. The phase of this amplitude modulation changes by $180^{\circ}$ from one side of a fringe to the other and vanishes at the top of each fringe. To convert the phase behavior of the modulation into a derivative shaped discriminator signal, the ac component of the detector signal is demodulated in a double balanced mixer whose local oscillator input is an appropriately phase shifted and amplified version of the $4.4 \mathrm{kHz} \mathrm{PZT}$ dither voltage.

The discriminator signal is used with proportional/ integral feedback to the PZT to control the length of the transfer cavity such that the frequency on the HeNe laser corresponds exactly to the center of the chosen transmission fringe. The $3 \mathrm{~dB}$ cutoff frequency of the servo loop is 
$f_{c}=1 / 2 \pi \tau=50 \mathrm{~Hz}$ with a unity gain frequency of $1.0 \mathrm{kHz}$. The maximum dc correction voltage that can be applied to the PZT is purposely limited to $\pm 11 \mathrm{~V}$ and yet is easily sufficient to lock the cavity. Hence the electronics can all be derived from conventional $\pm 15 \mathrm{~V}$ operational amplifiers and there is no need for a high voltage power supply. The $\pm 11 \mathrm{~V}$ limit translates into less than $1 / 3$ FSR change of the transmission frequency of the FPI in either direction. This is particularly relevant, since the fast PZT control can never cause a jump to another longitudinal mode of the transfer cavity, an event which would seriously debilitate the whole transfer stability concept.

To monitor the proper locking of the FPI two checks are performed. The first is to determine whether the residual error signal is within a predetermined range around 0 $\mathrm{V}$, which is monitored continuously via a front panel green/red light emitting diode (LED). More importantly, the dc part of the photodetector signal monitoring the transmitted light is compared to a level of about $1 / 3$ of its possible maximum at the top of a cavity fringe. If it drops below this limit, flip-flop circuitry is triggered which activates a red warning LED. The flip-flop can only be reset manually; hence the experimenter is alerted if the lock has ever been lost, and thus can have the assurance of complete lock integrily over an arbitrarily long series of spectral scans.

To counteract the effects of slow variations of the air pressure and temperature in the laboratory, the central stainless steel part of the cavity spacer is heated. The heater correction is derived from integral and proportional feedback of the discriminator signal, and added onto a constant $\mathrm{dc}$ current (the working point) to ensure an average temperature of about $20 \mathrm{~K}$ above ambient room temperature. This initial offset places the lock point more than 100 FSR away from that of a room temperature cavity, and hence yields both a large dynamic range and adequate speed for the heater control. The time constant of the proportional branch of the feedback is $1 \mathrm{~s}$ to separate it cleanly from the $50 \mathrm{~Hz}$ knee in the PZT control bandwidth. The corresponding integral part of the heater servo just serves to zero the two proportional corrections (i.e., to the PZT the heater), and for which a relatively long time constant of $100 \mathrm{~s}$ proves entirely sufficient. Furthermore, this time constant prevents rapid heating or cooling of the cavity during momentary interruptions of the lock (i.e., due to blockage of the HeNe laser beam) which could cause an undesired relocking to a different longitudinal mode of the transfer cavity.

\section{B. Frequency control of the $\mathrm{Ar}^{+}$laser}

For the frequency control of the single mode $\mathrm{Ar}^{+}$laser, the rear mirror is PZT mounted, with a voltage requirement of $435 \mathrm{~V}$ per FSR $(136 \mathrm{MHz})$ of the $110 \mathrm{~cm}$ laser cavity. The servo controller which stabilizes the output frequency of the $\mathrm{Ar}^{+}$laser, therefore, has to supply at least this range of travel so a cavity mode can always be found to match the desired frequency determined by one of the modes of the transfer cavity. Here we implicitly assume that the center frequency of the transmission bandpass of the $\mathrm{Ar}^{+}$intracavity etalon and the transfer cavity mode are separated by less than half the $\mathrm{Ar}^{+}$FSR. On the other hand, too great a dynamic range for the PZT could allow the frequency of a particular longitudinal mode of the $\mathrm{Ar}^{+}$ laser to tune by more than the $250 \mathrm{MHz}$ free spectral range of the transfer cavity. This would possibly lead to a situation where the servo loop controller could accidentally relock the $\mathrm{Ar}^{+}$laser to an adjacent mode of the FPI. A change of the $\mathrm{Ar}^{+}$laser frequency by exactly one FSR of the transfer cavity would result, which would therefore translate into a loss of the absolute frequency calibration of the setup. To prevent this, the controller is purposefully allowed less than $800 \mathrm{~V}$ travel, i.e., nearly 2 FSR of the $\mathrm{Ar}^{+}$laser but less than 1 FSR of the transfer cavity.

With these ideas in mind, an integral feedback with a time constant adjustable around $50 \mathrm{~ms}$ is used. It consists of a low voltage part followed by a high voltage amplifier $(\times 100)$. Since the transfer cavity is already dithered by the PZT, the necessary discriminator signal is derived from the $\mathrm{Ar}^{+}$light transmitted through the FPI in an analogous way to the $\mathrm{HeNe}$ laser. As the unavoidable thermal expansion or contraction of the $\mathrm{Ar}^{+}$laser cavity will eventually drive the controller into its lower or upper limit, provisions for an automatic reset are implemented, as described below. These also allow for the automatic startup of the system and initial locking to the transfer cavity.

As long as the light power transmitted through the FPI (dc part of the detector signal) is below a preset level, a small constant voltage is fed to the integrator, inducing a slow search for the proper lock point. As the transfer cavity fringe is approached, the detector signal rises, the discriminator signal is electronically switched to the integrator and the constant voltage disconnected, thereby quickly achieving a tight lock. The lock status is also indicated by a green/red LED on the front panel for operator convenience. If the PZT control voltage reaches either of the preset lower or upper limit, whose difference corresponds roughly to $1.5 \mathrm{FSR}$ of the $\mathrm{Ar}^{+}$laser cavity (typically 650 V), the integrator is reset to a suitable starting point, and the next adjacent lock point acquired after a short automated ramp search as described above. It should be emphasized that by this procedure a neighboring longitudinal mode $(N \rightarrow N \pm 1)$ of the $\mathrm{Ar}^{+}$lascr is locked to the same mode of the transfer cavity and therefore the frequency of this laser is kept constant throughout an arbitrary number of resets due to thermal expansion/contraction of the $\mathrm{Ar}^{+}$ cavity length.

\section{TEST OF THE PERFORMANCE}

\section{A. Optical and electronic tests}

The first performance test of the stabilization system comprises measurements of the residual error signals and of the $\mathrm{HeNe}$ and $\mathrm{Ar}^{+}$output frequencies. The residual error signal of the cavity lock onto the HeNe laser scales to a root-mean-square (rms) frequency error of less than 200 $\mathrm{kHz}$ between the $\mathrm{HeNe}$ laser and the cavity mode. In an independent experiment the beat note between the $\mathrm{HeNe}$ laser output and an $\mathrm{I}_{2}$ stabilized HeNe laser was measured, 
and demonstrated a long term stability on the order of 100 $\mathrm{kHz} / \mathrm{h}$, i.e., well within the published range. ${ }^{13}$ Therefore, the combined error due to the $\mathrm{HeNe}$ and the quality of the cavity lock is believed to be well below $1 \mathrm{MHz}$ and should not contribute noticeably to the overall system performance. In the absence of any serious external perturbations (such as accidental interruption of the beam), the same mode of the cavity stays easily locked to the $\mathrm{HeNe}$ for periods on the order of a week.

Locking the $\mathrm{Ar}^{+}$laser to the transfer cavity has a twofold effect. First, the long term frequency excursions clearly evident in the unstabilized laser are dramatically reduced and indeed are limited only by the long term stability of the transfer cavity. We did not find a simple way to measure this independently, but spectral scans of the IR difference frequency system over molecular transitions reported in Sec. IV B suffice to indicate an absolute stability of the $\mathrm{Ar}^{+}$laser of better than a few $\mathrm{MHz}$. Second, the linewidth of the laser is considerably decreased. This can both be measured from the residual error signal and independently (and thus more reliably) by observing the frequency distribution of the laser output with an additional scanning FPI. The latter test was performed with a confocal cavity of $2 \mathrm{GHz}$ free spectral range and a finesse of about 200 . Both measurements show that the linewidth of the laser is indeed reduced from the free running value of about $10 \mathrm{MHz}$ to typically $1 \mathrm{MHz}$. Since the best subDoppler linewidths we presently observe in the slit supersonic expansions are on the order of $30 \mathrm{MHz}$ (at 4000 $\mathrm{cm}^{-1}$ for Ar carrier gas), this improvement in frequency stability is nontrivial and should both increase the resolution and lead to more reliable line shape measurements.

\section{B. Spectroscopic measurements}

As a test of the overall performance of the stabilization and calibration setup described in this work, we have obtained repeated measurements of fixed molecular transitions of (HF) $)_{2}$ in the near IR. These spectra can be measured with sub-Doppler resolution and high signal-to-noise ratio in the difference frequency spectrometer, described elsewhere in detail. ${ }^{7}$ Briefly, tunable cw IR light is produced by difference frequency mixing of the $\mathrm{Ar}^{+}$laser light with the tunable output of the dye laser. The $(\mathrm{HF})_{2}$ clusters are formed by expanding a mixture of $\mathrm{Ar}$ and $\mathrm{HF}$ through a pulsed slit $(4 \mathrm{~cm} \times 100 \mu \mathrm{m})$ nozzle. Signal strength is further enhanced with a multipass White cell arrangement, yielding a net $64 \mathrm{~cm}$ absorption path length.

The $v_{\text {acc }}=1 \leftarrow 0 Q$ branch spectrum of $(\mathrm{HF})_{2}$ is scanned repetitively over a period of more than $6 \mathrm{~h}$. Representative samples of the resulting spectra are shown in Fig. 3. The wall clock time of data acquisition is shown for each spectrum. The displayed part is the ${ }^{R} Q_{0}^{-}$branch of the HF dimer free hydrogen stretch fundamental $\left(\nu_{\mathrm{acc}}\right)$ at $3893 \mathrm{~cm}^{-1} \cdot{ }^{15}$ The top trace is the transmission spectrum of the transfer cavity as the dye laser is scanned and which permits successive scans over the IR transitions to be quantitatively compared. The minor amplitude fluctuations on the dye laser fringes stems from residual frequency noise on the dye laser. At this level of spectral resolution, there

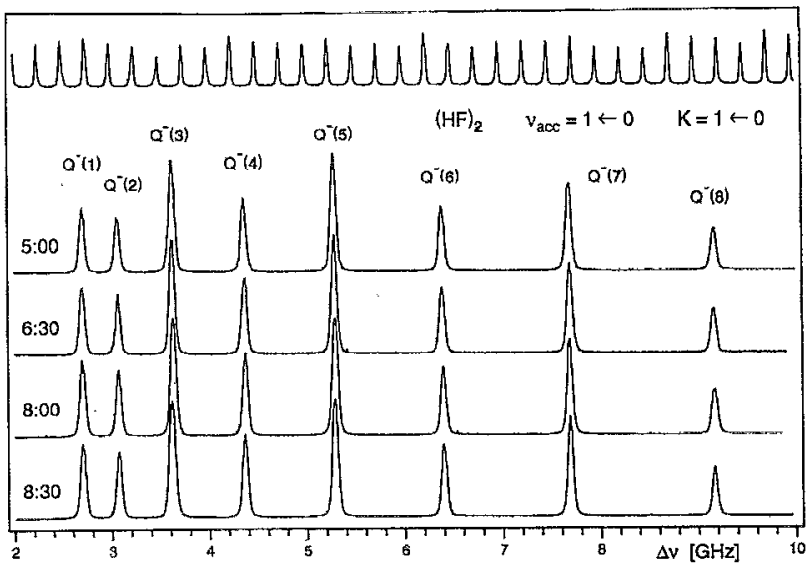

FIG. 3. Lower four traces: part of the ${ }^{R} Q_{0}^{-}$branch of the HF dimer free hydrogen stretch fundamental $\left(v_{\text {acc }}\right)$ at $3893 \mathrm{~cm}^{-1}$ recorded at subDoppler resolution. Upper trace: simultaneously recorded transmission fringes of the transfer cavity. The molecular spectrum was recorded various times (i.e., from 3:00 to 8:30 PM) to test the long term stability of calibration scheme described in this work.

is no noticeable shift between the spectra over the course of $6 \mathrm{~h}$, attesting to the long term frequency stability of the transfer cavity lock design. An even more exacting test of this frequency performance is described below.

For each of the experimental spectra a precise frequency scale is constructed in the following way: First the positions of all the cavity fringes are determined with a fast computer algorithm. ${ }^{16}$ Frequencies are then assigned to each of the fringes, and based on a linear scan between the fringes, also to each data point by interpolation. With this preliminary scale the position of the lines in the molecular spectrum are determined, and compared to the positions in the spectrum taken at 5:00 PM (i.e., arbitrarily chosen as a reference spectrum). Since the relative numbering of the dye laser cavity fringes must be determined from scan to scan, the fringe numbering is chosen visually by overlapping fringes and a given reference peak from successive scans. This procedure is completely justified since any drift of the overall system from scan to scan is certainly anticipated to be much less than 1 FSR $(\cong 250 \mathrm{MHz})$ and indeed is determined to be $\$ 5 \mathrm{MHz}$ over periods of up to a week. No adjustment is made for any drift of the $\mathrm{Ar}^{+}$laser frequency, i.e., it is locked solidly on the HeNe stabilized frequency transfer cavity and assumed to be constant.

With the frequency scale established for all the spectra, the position of each molecular line is determined and the difference between that and the position of the same line in the reference spectrum calculated. The set of differences for a given spectrum are averaged, and taken as a measure of the residual drift in the spectrometer. In addition, the standard deviation of the individual differences in a given spectrum are calculated to give a measure of the uncertainty of the frequency positions. The typical value of 5 $\mathrm{MHz}$ for the standard deviation in a spectrum with 30 $\mathrm{MHz}$ linewidths reflects the fact that the lines are not very smooth due to residual frequency fluctuations of the dye laser. This has been recently eliminated by locking the dye 


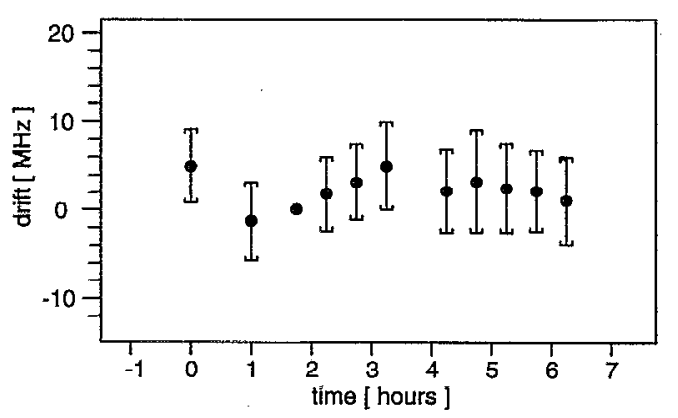

FIG. 4. Frequency drift of the difference frequency spectrometer as a function of relative time. The error bars represent \pm 1 standard deviation. The data scan taken at $+1.8 \mathrm{~h}$ is used as a reference. See text for details.

laser to a Zerodur cavity, which is scanned by an intracavity galvo plate.

The measured drifts over the series of $(\mathrm{HF})_{2}$ spectra are plotted as a function of the data acquisition time in Fig. 4. The error bars represent \pm 1 standard deviation. Within this error, no significant drift of the system over more than $6 \mathrm{~h}$ is apparent. We have made similar measurements ${ }^{17}$ under improved frequency control of the dye laser and have confirmed that this system exhibits a comparable degree of absolute frequency stability over the course of a full week. This indicates that an open transfer cavity locked to a stable reference laser is sufficient to calibrate sub-Doppler optical and IR molecular spectra with an accuracy on the order of a few $\mathrm{MHz}$ or better in routine, day to day applications.

\section{DISCUSSION}

A novel stabilization and calibration scheme for optical spectrometers has been presented, based on the stabilization of an open transfer cavity onto the output of a polarization stabilized HeNe laser. The $\mathrm{Ar}^{+}$and the dye laser used in our difference frequency spectrometer are referenced to this cavity. A calibration stability on the order of $1 \mathrm{MHz} / \mathrm{h}$ is experimentally demonstrated.

The setup presented avoids the difficulty in electronically separating the signals from the three laser beams that was discussed in a design by Coulombe and Pine. ${ }^{18}$ It has much higher precision than simple stabilizing schemes used for pulsed dye lasers. ${ }^{19}$ Furthermore, in contrast to an alternative frequency calibration based on the IR radiation ${ }^{20}$ the strong visible radiation is used for the servo control of the lasers and all of the IR light generated can be used for the actual experiment. In addition, there is no need for a highly sensitive electrooptic modulator in the reference cavity, as in an approach suggested by Bergmann and co-workers. ${ }^{21}$
Our scheme should also be useful for many other types of $\mathrm{cw}$ spectrometers and even for stabilization of the $\mathrm{cw}$ seed laser of an injection seeded high power Nd:YAG. The latter is highly desirable in applications such as Raman spectroscopy. ${ }^{22}$ If a reference laser exists with a stable output frequency close to the frequency of interest, then the residual drift due to air dispersion can be reduced even further. In any event, the present calibration drift rates of $1 \mathrm{MHz} / \mathrm{h}$ already provide an extremely useful tool for many applications of high resolution spectroscopy.

\section{ACKNOWLEDGMENTS}

We gratefully acknowledge funding from the National Science Foundation (CHE90-00641 and PHY90-12244). One of us (E. R.) was supported by the Deutsche Forschungsgemeinschaft through a research fellowship. The authors also particularly wish to acknowledge $\mathrm{H}$. Rohner and the late $\mathrm{R}$. Weppner for many fruitful discussions and the skillful implementation of the transfer cavity structure.

${ }^{1}$ E. Riedle, Th. Knittel, Th. Weber, and H. J. Neusser, J. Chem. Phys. 91, 4555 (1989); E. Riedle and H. J. Neusser, J. Chem. Phys. 80, 4686 (1984).

${ }^{2}$ W. A. Majewski and L. Meerts, J. Mol. Spectrosc. 104, 271 (1984).

${ }^{3}$ W. A. Majewski, D. F. Plusquellic, and D. W. Pratt, J. Chem. Phys. 90 , 1362 (1989).

${ }^{4}$ W. Demtröder, F. Paech, and R. Schmiedl, Chem. Phys. Lett. 26, 381 (1974).

${ }^{5}$ I. Burghardt, L. R. Zink, D. A. Fletcher, J. M. Brown, and I. R. Beattie, Mol. Phys. 67, 1401 (1989).

${ }^{6}$ A: McIlroy and D. J. Nesbitt, Chem. Phys. Lett. 187, 215 (1991).

${ }^{7}$ C. M. Lovejoy and D. J. Nesbitt, Rev. Sci. Instrum. 58, 807 (1987).

${ }^{8}$ A. H. Hielscher, C. E. Miller, D. C. Bayard, U. Simon, K. P. Smolka, R. F. Curl, and F. K. Tittel, Opt. Soc. Am. B 9, 1962 (1992);' P. Canarelli, Z. Benko, R. Curl, and F. K. Tittel, ibid. B 9, 197 (1992).

${ }^{9}$ J. H. Hall and S. A. Lee, Appl. Phys. Lett. 29, 367 (1976).

${ }^{10}$ J. Helmcke, J. J. Snyder, A. Morinaga, F. Mensing, and M. Gläser, Appl. Phys. B 43, 85 (1987).

${ }^{11}$ J. Hough, D. Hills, M. D. Rayman, L.-S. Ma, L. Hollberg, and J. L. Hall, Appl. Phys. B 33, 179 (1984).

${ }^{12}$ B. Edlen, Metrologia 2, 12 (1966).

${ }^{13}$ T. M. Niebauer, J. E. Faller, H. M. Goodwin, J. L. Hall, and R. L. Barger, Appl. Opt. 27, 1285 (1988).

${ }^{14}$ T. W. Hänsch and B. Couillaud, Opt. Commun. 35, 441 (1980).

${ }^{15}$ A. S. Pine and W. J. Lafferty, J. Chem. Phys. 78, 2154 (1983).

${ }^{16}$ J. J. Snyder, Appl. Opt. 19, 1223 (1980).

${ }^{17}$ M. A. Suhm, J. T. Farrell, Jr., S. H. Ashworth, and D. J. Nesbitt, J. Chem. Phys. 98, 5985 (1993).

${ }^{18}$ M. J. Coulombe and A. S. Pine, Appl. Opt. 18, 1505 (1979).

${ }^{19}$ K. G. Manohar, K. Dasgupta, B. M. Suri, and D. D. Bhawalkar, Rev. Sci. Instrum. 58, 920 (1987); K. Dasgupta and R. Srivastava, Appl. Opt. 26, 3659 (1987).

${ }^{20} \mathrm{~K}$. Sugiyama and J. Yoda, Opt. Commun. 95, 77 (1993).

${ }^{21}$ I. C. M. Littler, P. Jung, and K. Bergmann, Opt. Commun. 87, 61 (1992).

${ }^{22}$ J. A. Harrison, M. Zahedi, and J. W. Nibler, Opt. Lett. 18, 149 (1993). 